\title{
Growth and Chronic Disease
}

\author{
Leena Patel \\ University of Manchester, Central Manchester and Manchester Children's University Hospitals, Manchester, UK
}

\section{Key Words}

Acquired growth hormone resistance - Catch-up growth • Cystic fibrosis · Crohn's disease - Juvenile idiopathic arthritis • Delayed puberty • Growth failure/impairment • Skeletal growth · Somatic growth

\begin{abstract}
Growth impairment occurs with many chronic conditions. Cystic fibrosis, Crohn's disease and juvenile idiopathic arthritis are relatively common chronic diseases in childhood associated with substantial growth impairment. While growth failure may be the initial presenting feature of pathology, the pattern of growth is also a useful measure of disease severity and response to treatment. In addition to its diagnostic value, the resulting short stature cannot only be unacceptable to patients but can also have detrimental effects on the physical health of patients with conditions such as cystic fibrosis. Growth impairment in children with chronic disease is associated with disruption of the growth hormone (GH)insulin-like growth factor (IGF) axis, and predominantly results from undernutrition, chronic inflammation and prolonged corticosteroid treatment. Undernutrition leads to major adaptations in the endocrine system geared towards conserving energy, diverting substrates away from growth and reproduction, and providing alternative sources of energy for critical body homeostasis. Although chronic inflammatory processes exacerbate undernutrition, proinflammatory cytokines such as interleukin- 6 , tumor necrosis factor- $\alpha$ and interleukin-1 $\beta$ also adversely affect growth, independent of nutrition, by disrupting the integrity of the GH-IGF
\end{abstract}

C 2008 Nestec Ltd, Vevey/S. Karger AG, Basel 0517-8606/07/0653-0129\$23.50/0

ax +4161306 1234

E-Mail karger@karger.ch

www.karger.com
Accessible online at: www.karger.com/ane axis and by direct local effects on growth plate chondrogenesis. These growth-regulating mechanisms are disturbed further by corticosteroids used in some chronic conditions for their anti-inflammatory and immunosuppressive properties. It is likely that the growth-suppressing processes slow growth plate senescence, and this in turn enables catch-up growth on recovery from chronic disease and withdrawal of corticosteroid treatment. Trials of recombinant human $\mathrm{GH}$ (rhGH) in patients with growth failure suggest improvement in linear growth as well as beneficial effects on body composition and clinical course. These have been of relatively short duration, and long-term benefits as well as the safety of rhGH need to be evaluated particularly when given concomitantly with corticosteroids.

Copyright $\odot 2008$ Nestec Ltd., Vevey/S. Karger AG, Basel

\section{Introduction}

Chronic disease adversely affects growth, and the resulting impairment in height may be transient or sustained depending on the nature and course of the illness (table 1). The impairment in linear growth can be further exaggerated by delayed puberty which is commonly associated with chronic disease. Deviations from the normal pattern of growth may be the first clues to pathology as in celiac disease, Crohn's disease $[1,2]$ or renal tubular acidosis. In children known to have a chronic illness, serial records of height/length and weight plotted on standard distance charts are vital for monitoring wellbeing, disease activity, adverse clinical events and response to 
Table 1. Chronic conditions associated with growth impairment

Cystic fibrosis

Celiac disease

Crohn's disease

Chronic liver disease

Juvenile idiopathic arthritis

Chronic renal failure

Renal tubular acidosis

Congestive heart disease (large ventral septal defect)

Cyanotic heart disease (tetralogy of Fallot)

Human immunodeficiency virus infection

Asthma

Atopic dermatitis

Chronic hemolytic anemia (thalassemia major)

Long-term treatment with methylphenidate

treatment. Advances in management have altered the natural history of many conditions, reduced morbidity and improved survival. Where long-term remission of the primary disorder can be achieved, the outcome for growth and attainment of normal adult height are good. For children with refractory disease, impaired growth, short stature, delayed puberty and reduced adult height are increasingly highlighted as being problematic both physically and psychologically. This article presents an update on the general pathophysiology of impaired linear growth in chronic diseases and some key conditions in which substantial growth impairment occurs.

\section{Pathophysiology of Growth Impairment in Chronic Disease}

\section{Acquired Growth Hormone Resistance}

Growth in childhood is principally regulated by growth hormone $(\mathrm{GH})$ and insulin-like growth factors (IGFs). Impaired growth in children with chronic disease is associated with a state of relative GH resistance, reflected by normal or high spontaneous and stimulated serum GH, low IGF-1 and low IGF-binding protein-3 (IGFBP-3) levels [3, 4]. GH resistance is further illustrated by the relatively small increase in IGF-1 and IGFBP-3 levels (31 and 14\%, respectively) compared to age-matched controls (85 and 73\%) with the IGF-1 generation test in children with systemic juvenile idiopathic arthritis (JIA) [4]. The major factors disrupting the integrity of the $\mathrm{GH}-$ IGF-1 axis in children with chronic disease are undernutrition, chronic inflammation and corticosteroid treatment.

\section{Undernutrition}

Undernutrition is common in chronic diseases and results from multiple interrelated factors, such as anorexia, limited nutrient intake, malabsorption and unmet increased energy requirements. Prolonged lack of macronutrients leads to adaptive mechanisms geared towards conserving energy, diverting substrates away from growth and reproduction, and providing alternative sources of energy for critical body homeostasis. One consequence is relative GH resistance, and both IGF-1 and IGFBP-3 levels correlate with measures of nutrition in chronic illness [3]. Energy deficiency also has an inhibitory effect on the hypothalamic-pituitary-reproductive axis so that there is decreased pulsatile secretion of gonadotropins, possibly from reduced activity of the hypothalamic gonadotropin-releasing hormone pulse generator, and this accounts for the delay in pubertal development [5].

The key regulator of body weight and signal of energy stores is leptin and it is thought to be the modulator that links the nutritional state with neuroendocrine function at the level of the hypothalamus and pituitary [6]. In malnourished children, circulating leptin levels are low and correlate with low insulin as well as low IGF-1 concentrations [7]. In addition to an influence on the GH-IGF-1 axis and gonadotropin secretion, low circulating leptin may be the trigger that activates the hypothalamic-pituitary-adrenal axis and increases cortisol levels [8]. Along with low insulin and high GH levels, elevated levels of cortisol facilitate the switch from carbohydrate to fatbased metabolism by augmenting fatty acid mobilization, proteolysis and gluconeogenesis. Hypercortisolemia may also contribute to the growth impairment associated with nutritional deprivation by inhibiting IGF-1-dependent actions of GH [7]. These adaptive endocrine effects from chronic undernutrition are partly reversed by nutritional recovery but sustained weight gain is required for linear growth to improve.

\section{Chronic Inflammation}

Chronic inflammation underlies the pathophysiology of a number of chronic diseases. The tissue damage that ensues triggers a host of events including increased energy expenditure, fat mobilization, proteolysis, negative nitrogen balance, gluconeogenesis, anorexia and weight loss. Growth impairment occurs even when nutrition is adequately supplemented and highlights a direct modulatory effect of the inflammatory process on the integrity of the GH-IGF- 1 axis and the growth plate $[9,10]$. Studies in animal models have unraveled some of the mechanisms which underlie this [11-14]. 
Interleukin-6 (IL-6), tumor necrosis factor- $\alpha$ (TNF$\alpha$ ) and interleukin-1 $\beta$ (IL-1 $\beta$ ) play a key role in modifying the immune response to tissue injury and maintaining the chronic inflammatory response. In addition to high concentrations of these proinflammatory cytokines which correlate with disease activity $[15,16]$, levels of IGF-1 and IGFBP-3 are low in children with JIA [11], inflammatory bowel disease $[16,17]$, cystic fibrosis (CF) [3, 18].

Rats with experimental colitis demonstrate reduced linear growth in conjunction with increased concentrations of IL-6 [12] and significant improvements in growth when circulating IL- 6 is blocked by antibody [14]. Transgenic mice chronically overexpressing IL- 6 from birth also have markedly reduced growth and delayed puberty despite normal food intake [11]. Like children with chronic inflammation, these animals exhibit a state of GH resistance with normal or high GH levels and low levels of IGF-1 and IGFBP-3, and circulating IGF-1 and IGFBP-3 correlate inversely with IL-6 levels. These disease models suggest that low circulating IGFBP-3 results from IL-6induced proteases and increased proteolysis. While the corollary to this is shortened half-life and accelerated clearance of IGF-1, decreased levels of IGF-1 may also be due to reduced hepatic synthesis through downregulation of the GH receptor [11, 13]. As GH-binding protein is generated from proteolytic cleavage of the $\mathrm{GH}$ receptor, low levels of GHBP in GH-resistant states consequent to chronic inflammation provide surrogate evidence for a $\mathrm{GH}$ receptor defect in children [19]. By downregulating $\mathrm{GH}$-induced expression of the $\mathrm{GH}$ receptor gene in hepatocytes in vitro, IL-1 and TNF- $\alpha$ are also implicated in the dysregulation of the GH-IGF-1 system [20]. In addition, there is experimental evidence to suggest that these cytokines have local effects on the epiphyseal growth plate. IL- $1 \beta$ and TNF- $\alpha$ act in synergy to disrupt longitudinal bone growth in fetal rat metatarsals by inhibiting chondrocyte proliferation and inducing apoptosis, and these effects are partially reversed by IGF-1, anti IL-1 $\beta$ or anti-TNF- $\alpha$ [21].

\section{Corticosteroid Treatment}

The beneficial effects of corticosteroids as immunosuppressive and anti-inflammatory agents in the treatment of chronic diseases are accompanied by adverse affects on growth particularly when supra-physiological doses are administered long-term [22]. The mechanisms which lead to growth impairment are complex and include disruption of the GH-IGF axis at various levels, direct effects on the epiphyseal growth plate, and inhibi- tory effects on gonadotropin secretion and adrenal steroid production [23].

Treatment with recombinant human GH (rhGH; 43 $\mu \mathrm{g} / \mathrm{kg} / \mathrm{day}$ ) has the potential to partly reverse the growth retardation from corticosteroids [24]. Retrospective analysis of 83 patients enrolled in the National Cooperative Growth Study who had received corticosteroid treatment for at least 12 months (mean prednisone equivalent dose $0.5 \mathrm{mg} / \mathrm{kg} /$ day) revealed short stature (mean height standard deviation score, SDS, -3.7) and growth failure (mean height velocity $3 \mathrm{~cm} /$ year) at baseline [24]. rhGH treatment resulted in an increase in levels of IGF-1 and IGFBP3 , and height velocity in the first 2 years (mean $6.3 \mathrm{~cm} /$ year) [24]. Although only one patient in this cohort had transient hyperglycemia, rhGH in children on long-term corticosteroid treatment warrants careful evaluation owing to potential adverse effects on carbohydrate metabolism, immunostimulatory effects and oncogenic potential [24].

\section{Catch-Up Growth}

There is potential for catch-up growth if the underlying growth-inhibiting condition can be suppressed or modified. Catch-up is seen as accelerated linear growth and normalization of height towards parental target. It is described to be complete or incomplete depending on whether the final height attained is within or below the genetic potential, the latter defined by mid-parental target height range [25]. The extent to which growth is impaired and consequently the degree and duration of catch-up are determined by the nature of the chronic disease, age at onset, age at diagnosis, severity of disease activity and duration, nature and success of interventions, skeletal maturation and pubertal stage. In turn, adult height attained depends on the severity of growth retardation during the active phase of the disease, the extent of catch-up growth after remission, and the timing and progression of puberty.

Catch-up growth is thought to be due to changes in the epiphyseal growth plate [26]. Linear growth is the result of endochondral ossification of long bones and vertebrae when cartilage is formed and remodeled into bone at the growth plate. Chondrogenesis comprises proliferation and hypertrophy of the growth plate chondrocytes and synthesis of cartilaginous matrix. It is regulated by systemic endocrine as well as local paracrine growth factors and IGF-1 acts as both [27]. With age, growth rate slows in conjunction with functional and structural changes in the growth plate. There is a steady decrease in the rate of chondrocyte proliferation, number of chondrocytes and 
height of the growth plate, and linear growth finally stops with ossification of the growth plate [28]. This decline in growth rate is likely to be due to programmed mechanisms intrinsic to the growth plate and has been referred to as 'growth plate senescence' [29]. Recent work in rabbits suggests that chondrocytes in the resting zone of the growth plate serve as a pool of stem-like cells, and over time the number of cells as well as their proliferative capacity gradually decline [30]. Chronic disease, inflammation and corticosteroid treatment suppress the depletion and proliferation of cells, and thereby conserve the population and proliferative capacity of resting zone chondrocytes. Thus growth plate senescence is slowed and this allows for catch-up growth when the growth-inhibiting conditions are removed [26]. As bone age represents skeletal maturation, is considered a surrogate marker for growth plate senescence and is inversely related to linear growth potential, delayed bone age is a good predictor of catch-up growth [26].

\section{Specific Chronic Diseases Associated with Substantial Growth Impairment}

\section{Cystic Fibrosis}

$\mathrm{CF}$ is the commonest autosomal recessive condition in the Western world. It is a chronic progressive multisystem disease affecting exocrine glands, primarily the respiratory tract, pancreas and sweat glands. Patients have chronic respiratory infections and inflammation, pancreatic insufficiency, restricted quality of life and reduced life expectancy. Undernutrition results from malabsorption, anorexia, inadequate intake and increased requirements. Poor weight gain and linear growth occur from birth until the time of diagnosis, and failure to thrive is one of the classical presenting features of $\mathrm{CF}$ after the neonatal period [31]. The National Cystic Fibrosis Patient registry data revealed that approximately $40 \%$ of infants were below the 5 th percentile for weight and length at diagnosis [31]. Owing to some catch-up growth associated with treatment interventions following diagnosis, a smaller proportion (20-25\%) had weight and length below the 5 th percentile at 2 years of age. However, despite aggressive management, patients with CF continue to have deficits in growth throughout childhood [31, 32]. In addition the growth impairment is more severe during adolescence owing to the progressive nature of the disease and increased morbidity with age. This is associated with a delay in skeletal maturation of up to 24 months and in onset of puberty in some patients [32]. Delayed timing of the pubertal growth spurt is illustrated by a delay in age at peak height velocity by $9-10$ months in male and 10-14 months in female patients [33, 34]. The greater the delay, the lower the peak height velocity, and patients with an extreme delay in puberty fail to demonstrate a significant growth spurt [33]. Thus adult height is significantly compromised and a cross-sectional survey of male and female patients in the UK found it was -1 and -0.5 SDS, respectively, below the reference population [32].

Aggressive nutritional intervention with a high fat, high calorie diet and a higher dose of pancreatic enzyme treatment has positive effects on growth and survival but is not sufficient to completely normalize weight and height [35]. In addition, despite failing to reach the critical body fat of $17 \%$ and weight of $47.8 \mathrm{~kg}, 20 \%$ of girls with $\mathrm{CF}$ achieve menarche $[33,34,36]$. These observations highlight that undernutrition is not the sole factor which contributes to impaired growth and delayed puberty in patients with CF. The impact of undernutrition and chronic inflammation on growth is further determined by age and mode of diagnosis, presentation with meconium ileus and need for surgery, nature of the therapeutic interventions (e.g. oral/inhaled corticosteroid treatment and nutritional management), and additional morbidity such as insulin deficiency and liver dysfunction. Children diagnosed early from neonatal screening have better growth than those diagnosed by a positive family history or clinical features of CF (mean age at diagnosis 13 vs. 100 weeks) [37]. In comparison to children diagnosed through neonatal screening before 12 weeks age and prior to the development of symptoms, those with meconium ileus necessitating surgery have significant growth impairment from birth with mean weight and height across all ages around the 30th percentiles [38].

Serial measurements in individual children with CF illustrate the longitudinal variations in growth and that periods of weight loss and growth failure are closely associated with adverse clinical events (mainly respiratory exacerbations and gastrointestinal complications) [39]. As might be expected, the severity of lung disease and airway colonization with Pseudomonas aeruginosa have a negative influence on growth and onset of puberty [33]. The observations of a relationship between plasma insulin area under curve (intravenous glucose tolerance test) and height velocity as well as serum IGFBP-3 suggests that declining insulin secretion is another factor which adversely affects growth by disturbing the GH-IGF-1 axis [40]. 
In patients with $\mathrm{CF}$, weight gain and stature are closely related to lung function [41], and inadequate weight gain and poor linear growth have major repercussions on physical health, survival and effectiveness of treatment. In the context of GH resistance in these patients, the anabolic and growth-promoting effects of recombinant human IGF-1 (rhIGF-1) and rhGH treatment have been explored. One placebo-controlled trial of rhIGF-1 treatment for 6 months in 7 CF patients found an increase in IGF-1 levels but no effects on linear growth and weight gain with treatment [42]. Its tendency to induce hypoglycemia might be beneficial in patients with impaired glucose tolerance but detrimental when glucose homeostasis is normal.

rhGH treatment in children with CF has the potential to enhance linear growth and weight gain, and thereby improve lung function and slow disease progression. Indeed improvements in weight and height in prepubertal children with CF have been reported from randomized trials of rhGH treatment for up to 2 years [43-45]. The first randomized controlled trial over 12 months in 19 prepubertal patients, whose initial height and weight were $\leq 10$ th percentile despite adequate calorie intake, documented greater height velocity ( 8.1 vs. $3.8 \mathrm{~cm} /$ year), weight velocity ( 4.5 vs. $2.1 \mathrm{~kg} /$ year) and an increase in lean mass ( $4.7 \mathrm{vs.} 2.1 \mathrm{~kg}$ ) in those who received rhGH 0.43 $\mu \mathrm{g} / \mathrm{kg} / \mathrm{day}$ [43]. Comparable results were reported from randomized cross-over trials with rhGH for 6 months in 10 children [45] and for 12 months in 61 patients [44]. Pubertal adolescents studied retrospectively ( 25 patients, of whom 13 were given rhGH $0.43-0.5 \mu \mathrm{g} / \mathrm{kg} /$ day for 12 months) also had better growth in height and weight with treatment [46]. The improvements in growth and lean mass with rhGH may be due to improved whole body protein catabolism and improved efficiency of whole body protein kinetics [43]. Although there was improved exercise tolerance [45], fewer hospitalizations [44, 46] and reduced outpatient intravenous antibiotic courses [43] with rhGH, the effects on lung function and clinical state in these studies are not consistent and may be explained by the relatively short duration of rhGH treatment as well as differences in management of patients at the various centers. None of the patients in these trials developed diabetes mellitus during treatment with $\operatorname{rhGH}[43,45]$. Long-term studies should provide evidence for the potential benefits of rhGH treatment on lung disease, body composition, bone density, adult height, quality of life and survival, as well as cost and safety, particularly for glucose tolerance.

Growth and Chronic Disease

\section{Crohn's Disease}

Crohn's disease and ulcerative colitis are the two most common forms of chronic inflammatory bowel disease in children. Inflammation commonly affects the small and/or large intestine, but can affect the entire gastrointestinal tract in Crohn's disease and is restricted to the large intestine in ulcerative colitis. Classical symptoms are chronic diarrhea, abdominal pain and weight loss, but the diarrhea is classically bloody in ulcerative colitis. Reduced height and weight velocities are common features of both conditions in the year preceding diagnosis. After diagnosis and initiation of treatment, growth impairment is common in children with Crohn's disease but unusual in ulcerative colitis [2, 47]. A large populationbased series of children with Crohn's disease and ulcerative colitis found height SDSs of -0.5 and -0.1 , respectively, and height was less than the 3rd percentile for 13 and 3\%, respectively, at diagnosis [46]. In Crohn's disease, growth failure may precede intestinal symptoms as well as weight loss and its pathological significance as the sole initial feature in children should not be undermined [1]. Delay in onset and progression of puberty are frequently seen particularly in prepubertal patients with active disease or frequent relapses.

The deficits in growth and delay in puberty are not fully reversed by adequate calorie intake. In addition to undernutrition, uncontrolled intestinal inflammation is important in the etiology of growth impairment and other contributing factors are duration of symptoms before diagnosis and thus delay in diagnosis, disease severity and location particularly in the jejunum [2, 47]. Deficits in attained adult height are seen in up to a third of the patients [48] and have been found to be related to height at diagnosis as well as the presence of jejunal disease [48]. Although polymorphisms in the NOD2 gene are independent risk factors for susceptibility to Crohn's disease and are associated with disease severity, ileal involvement and the need for surgery, they do not appear to have a direct link with growth impairment [49].

Treatment strategies aimed to suppress inflammation and improve nutritional status include enteral nutrition with polymeric diet for widespread disease, intestinal resection for localized disease and monoclonal anti-TNF- $\alpha$ antibody infliximab. They result in increased circulating levels of IGF-1 and IGFBP-3 [17, 50, 51] and an acceleration in linear growth $[17,52]$. Catch-up growth and improvement in final height are likely if the interventions are prior to or in early puberty [52].

There has been some attempt to investigate the potential benefits of rhGH treatment to reverse the catabolic 
state of active Crohn's disease, enhance mucosal healing and improve linear growth [53]. While rhGH treatment $(50 \mu \mathrm{g} / \mathrm{kg} /$ day $)$ was associated with an increase in IGF-1 and IGFBP-3 levels over a brief duration of up to 2 years, no firm conclusions can be drawn from the small number of children studied.

\section{Juvenile Idiopathic Arthritis}

JIA replaces the previously used terms juvenile chronic arthritis and juvenile rheumatoid arthritis, and includes heterogenous forms of chronic arthritis of unknown cause that begin before the age of 16 years and persist for more than 6 weeks [54]. The subtypes classified under JIA (systemic, oligo-, rheumatoid factor-positive poly-, rheumatoid factor-negative poly-, enthesitisrelated, psoriatic and undifferentiated arthritis) have distinct features and varying spectrums of disease severity. Although uncommon at presentation, growth impairment is evident within a few years in patients with unremitting disease and is influenced by the duration of disease [55]. Deficits in height are greater in patients with systemic and rheumatoid factor-positive polyarticular arthritis than oligo- and rheumatoid factor-negative arthritis $[55,56]$. Delayed puberty further compromises linear growth in these patients and greater growth impairment is observed during the pubertal years compared to before puberty (mean height SDS -0.71 vs. -0.1 ) [56].

In addition to disease subtype, course and duration, corticosteroid treatment further impairs growth and the onset of puberty in JIA. A mean loss of height of -2.7 SDS has been observed in the first year of the disease in patients receiving prednisone [57]. The adverse effect on height is influenced by the treatment duration especially if it is longer than 12 months. On discontinuing treatment, 70\% showed some catch-up growth and although the mean adult height in this subgroup was better than in those without catch-up ( -1.5 SDS compared to -3.6 SDS), a significant impairment persisted [57]. Overall, final height was less than -2 SDS in $40 \%$ of patients and below the target height in over 80\% [57].

Trials of rhGH in selected children with persistently active JIA and severe growth failure have reported improvements in linear growth after 6 months of treatment [58] as well as a sustained effect for up to 4 years $[59,60]$. Intervening with rhGH earlier on in the course of the disease is speculated to minimize the significant loss in height, especially in patients treated with corticosteroids [59]. A significant increase in height velocity compared to baseline but no change in height SDS were observed in the first 2 years in an open uncontrolled study in 13 ex- ceptionally short corticosteroid-treated patients (mean height -4.6 SDS) [59]. Additional benefits of rhGH proposed from this study were an increase in lean mass and lumbar bone mineral density. In a recent study of 38 prepubertal children with severe systemic or polyarticular disease and treated with corticosteroids, 6 were identified to have GHD [60]. The 12 non-GHD patients treated with $\mathrm{rhGH}(47 \mu \mathrm{g} / \mathrm{kg} / \mathrm{day})$ were observed to have increase in IGF-1 and IGFBP-3 levels, height velocity and height SDS during 4 years of treatment (mean height SDS increased from -2.8 to -1.9 compared to decline from -2.3 to -3.0 SDS in the untreated patients) [60]. Whereas disease activity and the dose of prednisolone have a negative effect, the dose of rhGH has a positive effect on the growth response [60]. Although no adverse effects were observed in these patients, transient glucose intolerance with rhGH reported in a previous study highlights the need to evaluate safety long-term [59].

\section{Effects on Growth of Anti-TNF- $\alpha$ Treatment}

For Crohn's disease and JIA refractory to conventional treatment, anti-TNF- $\alpha$ agents can induce and maintain remission and reduce the need for corticosteroids. In addition to dampening the inflammatory process, these agents are also postulated to counteract the modulatory effect of proinflammatory cytokines on the GH-IGH-1 axis and to thereby enhance linear growth. Changes in linear growth have been described in an observational study of 32 children (age 4.7-17.3 years and mean height -0.4 SDS at diagnosis) with refractory Crohn's disease given infliximab. The mean height SDS prior to this treatment was -1.1 SDS. After a median of 26 months of infliximab treatment, prepubertal and early pubertal patients had a greater increase in mean height SDS compared to those in advanced stages of puberty ( +0.5 vs. +0.2 SDS) [52]. Increased height velocity in the 1st year (mean 7.6 vs. $3.7 \mathrm{~cm}$ before treatment), catch-up growth after 2 years (mean height SDS -1.1 vs. -2.4 before treatment) and a significant increase in serum IGF-1 and IGFBP-3 levels have been reported from a study of $18 \mathrm{pa}$ tients with refractory JIA given etanercept [61]. A further study in 71 patients (mean age 9.6 years, mean duration of JIA 5.7 years) suggested growth failure before the intervention was the strongest predictor of catch-up growth during 2 years of treatment with etanercept or infliximab [62]. While such anti-inflammatory treatments hold great promise for patients in the future in concurrently modifying the disease process, reducing associated morbidity and improving growth outcome, their long-term benefits and risks need to be explored. 


\section{Conclusion}

Studies over the last decade have provided greater understanding of the etiology of growth impairment in chronic disease, in particular the molecular mechanisms which disrupt the integrity of the GH-IGF axis and cellular changes in the epiphyseal growth plate. Although advances in management have altered the natural history of many conditions, reduced morbidity and improved survival, significant growth impairment is still seen in patients with conditions such as CF, Crohn's disease and JIA. This can have detrimental effects on physical health, especially for patients with CF. Along with delayed puberty and the potential for short stature in adulthood, growth impairment is also a psychological burden for patients with chronic disease. A treatment which has the potential to optimize growth in this context is rhGH. Trials of rhGH in selected patients with growth failure are thus far limited by the relatively short duration of treatment but suggest improvements in linear growth as well as beneficial effects on body composition and clinical course. The long-term safety of rhGH needs to be evaluated particularly when given concomitantly with corticosteroids. Although not yet extensively studied, new antiTNF agents hold great promise in concurrently modifying the inflammatory process, reducing associated morbidity and improving growth outcome for patients with refractory Crohn's disease and JIA.

\section{References}

>1 Kanof ME, Lake AM, Bayless TM: Decreased height velocity in children and adolescents before the diagnosis of Crohn's disease. Gastroenterology 1988;95:1523-1527.

$\checkmark 2$ Motil KJ, Grand RJ, Davis-Kraft L, et al: Growth failure in children with inflammatory bowel disease: a prospective study. Gastroenterology 1993;105:681-691.

3 Taylor AM, Bush A, Thomson A, et al: Relation between insulin-like growth factor-I, body mass index, and clinical status in cystic fibrosis. Arch Dis Child 1997;76:304-309.

4 Tsatsoulis A, Siamopoulou A, Petsoukis C, et al: Study of growth hormone secretion and action in growth-retarded children with juvenile chronic arthritis (JCA). Growth Horm IGF Res 1999;9:143-149.

5 Cunningham MJ, Clifton DK, Steiner RA: Leptin's actions on the reproductive axis: perspectives and mechanisms. Biol Reprod 1999;60:616-622

-6 Yu WH, Kimura M, Walczewska A, et al Role of leptin in hypothalamic-pituitary function. Proc Natl Acad Sci USA 1997;94: 1023-1028.

7 Soliman AT, El Zalabany MM, Salama M, Ansari BM: Serum leptin concentrations during severe protein-energy malnutrition: correlation with growth parameters and endocrine function. Metabolism 2000;49:819825 .

$>8$ Licinio J, Masntzoros C, Negarao AB: Human leptin levels are pulsatile and inversely related to pituitary-adrenal function. Nat Med 1997;3:573-579.

$\checkmark 9$ Ballinger AB, Azooz O, El-Haj T, et al: Growth failure occurs through a decrease in insulin-like growth factor 1 which is independent of undernutrition in a rat model of colitis. Gut 2000;46:694-700.
10 Wong SC, Macrae VE, McGrogan P, Ahmed SF: The role of pro-inflammatory cytokines in inflammatory bowel disease growth retardation. J Pediatr Gastroenterol Nutr 2006; 43:144-155.

11 De Benedetti F, Meazza C, Oliveri M, et al: Effect of IL-6 on IGF binding protein-3: a study in IL- 6 transgenic mice and in patients with systemic juvenile idiopathic arthritis. Endocrinology 2001;142:4818-4826.

12 Koniaris SG, Fisher SE, Rubin CT, Chawla A: Experimental colitis impairs linear growth independent of nutritional factors. J Paediatr Gastroenterol Nutr 1997;25:137-141.

13 Lieskovska J, Guo D, Derman E: IL-6-overexpression brings about growth impairment potentially through a $\mathrm{GH}$ receptor defect. Growth Horm IGF Res 2002;12:388-398.

14 Sawczenko A, Azooz O, Paraszczuk J, et al: Intestinal inflammation-induced growth retardation acts through IL- 6 in rats and depends on the -174 IL-6 G/C polymorphism in children. Proc Natl Acad Sci USA 2005; 102:13260-13265.

15 Borrelli O, Bascietto C, Viola F, et al: Infliximab heals intestinal inflammatory lesions and restores growth in children with Crohn's disease. Dig Liver Dis 2004;36:342-347.

16 Street ME, de'Angelis G, Camacho-Hubner C, et al: Relationships between serum IGF-1, IGFBP-2, interleukin-1beta and interleukin6 in inflammatory bowel disease. Horm Res 2004;61:159-164.

17 Beattie RM, Camacho-Hubner C, Wacharasindhu $\mathrm{S}$, et al: Responsiveness of IGF-I and IGFBP-3 to therapeutic intervention in children and adolescents with Crohn's disease. Clin Endocrinol (Oxf) 1998;49:483489.
18 Street ME, Ziveri MA, Spaggiari C, et al: Inflammation is a modulator of the insulinlike growth factor (IGF)/IGF-binding protein system inducing reduced bioactivity of IGFs in cystic fibrosis. Eur J Endocrinol 2006; 154:47-52.

19 Postel-Vinay MC, Tar A, Crosnier H, et al: Plasma growth hormone-binding activity is low in uraemic children. Pediatr Nephrol 1991;5:545-547.

20 Thissen JP, Verniers J: Inhibition by interleukin- $1 \beta$ and tumour necrosis factor- $\alpha$ of the insulin-like growth factor I messenger ribonucleic acid response to growth hormone in rat hepatocyte primary culture. Endocrinology 1997;138:1078-1084.

21 Martensson K, Chrysis D, Savendahl L: Interleukin-1beta and TNF-alpha act in synergy to inhibit longitudinal growth in fetal rat metatarsal bones. J Bone Miner Res 2004; 19:1805-1812.

22 Hyams JS, Carey DE: Corticosteroids and growth. J Pediatr 1988;113:249-254.

23 Hochberg Z: Mechanisms of steroid impairment of growth. Horm Res 2002;58(suppl 1): 33-38.

24 Allen DB, Julius JR, Breen TJ, Attie KM: Treatment of glucocorticoid-induced growth suppression with growth hormone. National Cooperative Growth Study. J Clin Endocrinol Metab 1998;83:2824-2829.

25 Wit JM, Boersma B: Catch-up growth: definition, mechanisms, and models. J Pediatr Endocrinol Metab 2002;15:1229-1241.

26 Emons JA, Boersma B, Baron J, Wit JM: Catch-up growth: testing the hypothesis of delayed growth plate senescence in humans. J Pediatr 2005;147:843-846.

$\checkmark 27$ De Luca F: Impaired growth plate chondrogenesis in children with chronic illnesses. Pediatr Res 2006;59:625-629. 
28 Nilsson O, Baron J: Impact of growth plate senescence on catch-up growth and epiphyseal fusion. Pediatr Nephrol 2005;20:319322.

-29 Baron J, Klein KO, Colli MJ, et al: Catch-up growth after glucocorticoid excess: a mechanism intrinsic to the growth plate. Endocrinology 1994;135:1367-1371.

-30 Schrier L, Ferns SP, Barnes KM, et al: Depletion of resting zone chondrocytes during growth plate senescence. J Endocrinol 2006; 189:27-36.

$>31$ Lai HC, Kosorok MR, Sondel SA, et al: Growth status in children with cystic fibrosis based on the National Cystic Fibrosis Patient Registry data: evaluation of various criteria used to identify malnutrition. J Pediatr 1998;132:478-485.

32 Morison S, Dodge JA, Cole TJ, et al; UK Cystic Fibrosis Survey Management Committee: Height and weight in cystic fibrosis: a cross sectional study. Arch Dis Child 1997;77:497500.

>33 Byard PJ: The adolescent growth spurt in children with cystic fibrosis. Ann Hum Biol 1994;21:229-240.

-34 Johannesson M, Gottlieb C, Hjelte L: Delayed puberty in girls with cystic fibrosis despite good clinical status. Pediatrics 1997;99: 29-34.

>35 Corey M, McLaughlin FJ, Williams M, Levison $\mathrm{H}$ : A comparison of survival, growth and pulmonary function in patients with cystic fibrosis in Boston and Toronto. J Clin Epidemiol 1988;41:583-591.

-36 Frisch RE, Revelle R: Height and weight at menarche and a hypothesis of menarche. Arch Dis Child 1971;46:695-701.

\37 Farrell PM, Kosorok MR, Rock MJ, et al; Wisconsin Cystic Fibrosis Neonatal Screening Group: Early diagnosis of cystic fibrosis through neonatal screening prevents severe malnutrition and improves long-term growth. Pediatrics 2001;107:1-13.

$\checkmark 38$ Lai HC, Kosorok MR, Laxova A, et al: Nutritional status of patients with cystic fibrosis with meconium ileus: a comparison with patients without meconium ileus and diagnosed early through neonatal screening. Pediatrics 2000;105:53-61.

39 Greco L, Santamaria F, Salvatore D, de Ritis G: Growth dynamics in cystic fibrosis. Acta Paediatr 1993;82:254-260.
40 Ripa P, Robertson I, Cowley D, et al: The relationship between insulin secretion, the insulin-like growth factor axis and growth in children with cystic fibrosis. Clin Endocrinol 2002;56:383-389.

41 Zemel BS, Jawad AF, FitzSimmons S, Stallings VA: Longitudinal relationship among growth, nutritional status, and pulmonary function in children with cystic fibrosis: analysis of the Cystic Fibrosis Foundation National CF Patient Registry. J Pediatr 2000; 137:374-380.

42 Bucuvalas JC, Chernausek SD, Alfaro MP, et al: Effect of insulinlike growth factor-1 treatment in children with cystic fibrosis. J Pediatr Gastroenterol Nutr 2001;33:576-581.

43 Hardin DS, Ellis KJ, Dyson M, et al: Growth hormone improves clinical status in prepubertal children with cystic fibrosis: results of a randomized controlled trial. J Pediatr 2001; 139:636-642.

44 Hardin DS, Adams-Huet B, Brown D, et al: Growth hormone treatment improves growth and clinical status in prepubertal children with cystic fibrosis: results of a multicenter randomized controlled trial. J Clin Endocrinol Metab 2006;91:4925-4929.

45 Hutler M, Schnabel D, Staab D, et al: Effect of growth hormone on exercise tolerance in children with cystic fibrosis. Med Sci Sports Exerc 2002;34:567-572.

46 Hardin DS, Rice J, Ahn C, et al: Growth hormone treatment enhances nutrition and growth in children with cystic fibrosis receiving enteral nutrition. J Pediatr 2005;146: 324-328.

47 Sawczenko A, Sandhu BK: Presenting features of inflammatory bowel disease in Great Britain and Ireland. Arch Dis Child 2003;88: 995-1000.

48 Sawczenko A, Ballinger AB, Savage MO, Sanderson IR: Clinical features affecting final adult height in patients with pediatriconset Crohn's disease. Pediatrics 2006;118: 124-129.

49 Wine E, Reif SS, Leshinsky-Silver E, et al: Pediatric Crohn's disease and growth retardation: the role of genotype, phenotype, and disease severity. Pediatrics 2004;114:12811286

50 Bannerjee K, Camacho-Hubner C, Babinska $\mathrm{K}$, et al: Anti-inflammatory and growthstimulating effects precede nutritional restitution during enteral feeding in Crohn disease. J Pediatr Gastroenterol Nutr 2004;38: $270-275$.
51 Vespasiani Gentilucci U, Caviglia R, Picardi A, et al: Infliximab reverse growth hormone resistance associated with inflammatory bowel disease. Aliment Pharmacol Ther 2005;21:1063-1071.

52 Walters TD, Gilman AR, Griffiths AM: Linear growth improves during infliximab therapy in children with chronically active severe Crohn's disease. Inflamm Bowel Dis 2007;13:424-430.

53 Calenda KA, Schornagel IL, Sadeghi-Nejad A, Grand RJ: Effect of recombinant growth hormone treatment on children with Crohn's disease and short stature: a pilot study. Inflamm Bowel Dis 2005;11:435-441.

54 Ravelli A, Martini A: Juvenile idiopathic arthritis. Lancet 2007;369:767-778.

55 Liem JJ, Rosenberg AM: Growth patterns in juvenile rheumatoid arthritis. Clin Exp Rheumatol 2003;21:663-668.

56 Polito C, Strano CG, Olivieri AN, et al: Growth retardation in non-steroid treated juvenile rheumatoid arthritis. Scand J Rheumatol 1997;26:99-103.

-57 Simon D, Fernando C, Czernichow P, Prieur AM: Linear growth and final height in patients with systemic juvenile idiopathic arthritis treated with longterm glucocorticoids. J Rheumatol 2002;29:1296-1300.

58 Saha MT, Haapasaari J, Hannula S, et al: Growth hormone is effective in the treatment of severe growth retardation in children with juvenile chronic arthritis. Double blind placebo-controlled followup study. J Rheumatol 2004;31:1413-1417.

59 Simon D, Lucidarme N, Prieur AM, et al: Effects on growth and body composition of growth hormone treatment in children with juvenile idiopathic arthritis requiring steroid therapy. J Rheumatol 2003;30:24922499.

60 Bechtold S, Ripperger P, Hafner R, et al: Growth hormone improves height in patients with juvenile idiopathic arthritis: 4year data of a controlled study. J Pediatr 2003;143:512-519.

61 Schmeling H, Seliger E, Horneff G: Growth reconstitution in juvenile idiopathic arthritis treated with etanercept. Clin Exp Rheumatol 2003;21:779-784.

62 Tynjala P, Lahdenne P, Vahasalo P, et al: Impact of anti-TNF treatment on growth in severe juvenile idiopathic arthritis. Ann Rheum Dis 2006;65:1044-1049. 